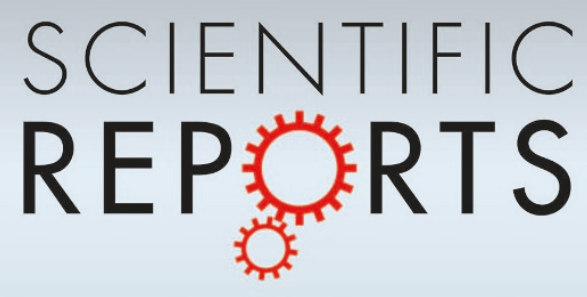

OPEN

SUBJECT AREAS:

NANOPARTICLES

MAGNETIC PROPERTIES AND

MATERIALS

Received

21 March 2014

Accepted

2 July 2014

Published

21 July 2014

Correspondence and requests for materials should be addressed to

D.S. (schmitz@

helmholtz-berlin.de)

* Current address: Peter Grünberg Institut

(PGI-6),

Forschungszentrum Jülich, 52425 Jülich,

Germany.

$\uparrow$ Current address: Department of Physics and Astronomy,

Vanderbilt University, Station B \#351807, 2301 Vanderbilt Place, Nashville, TN 37235-1807, USA.

\section{The dipole moment of the spin density as a local indicator for phase transitions}

\author{
D. Schmitz ${ }^{1}$, C. Schmitz-Antoniak ${ }^{2 *}$, A. Warland ${ }^{2}$, M. Darbandi ${ }^{2} \uparrow$, S. Haldar ${ }^{3}$, S. Bhandary ${ }^{3}$, O. Eriksson ${ }^{3}$, \\ B. Sanyal ${ }^{3} \& H$. Wende ${ }^{2}$
}

${ }^{1}$ Helmholtz-Zentrum Berlin für Materialien und Energie, Albert-Einstein-Straße 15, D-12489 Berlin, Germany, ${ }^{2}$ Fakultät für Physik and Center for Nanointegration Duisburg-Essen (CENIDE), Universität Duisburg-Essen, Lotharstr. 1, D-47048 Duisburg, Germany, ${ }^{3}$ Division of Materials Theory, Department of Physics and Astronomy, Uppsala University, Box-516, SE 75120 Uppsala, Sweden.

\begin{abstract}
The intra-atomic magnetic dipole moment - frequently called $\left\langle T_{z}\right\rangle$ term - plays an important role in the determination of spin magnetic moments by $\mathrm{x}$-ray absorption spectroscopy for systems with nonspherical spin density distributions. In this work, we present the dipole moment as a sensitive monitor to changes in the electronic structure in the vicinity of a phase transiton. In particular, we studied the dipole moment at the $\mathrm{Fe}^{2+}$ and $\mathrm{Fe}^{3+}$ sites of magnetite as an indicator for the Verwey transition by a combination of $\mathrm{x}$-ray magnetic circular dichroism and density functional theory. Our experimental results prove that there exists a local change in the electronic structure at temperatures above the Verwey transition correlated to the known spin reorientation. Furthermore, it is shown that measurement of the dipole moment is a powerful tool to observe this transition in small magnetite nanoparticles for which it is usually screened by blocking effects in classical magnetometry.
\end{abstract}

$\gamma$ -ray absorption near edge spectroscopy (XANES) and its associated magnetic circular dichroism (XMCD) is an experimental technique that allows for the determinination of magnetic properties element-specifically. Today, it is widely spread as one main advantage of XMCD that the orbital moment and the spin moment can be determined independently from each other using sum rules ${ }^{1,2}$ which have been experimentally confirmed for the $3 d$ transition metals ${ }^{3}$. The intra-atomic magnetic dipole moment of the $3 d$ spin-density distribution is one term in the XMCD sum rule for the spin moment. More precisely, with the spin sum rule the effective spin moment $\left(\mu_{S, e f f}\right)$, which is the sum of the intrinsic spin moment $\left(-2\left\langle S_{z}\right\rangle \mu_{B}\right)$ and the dipole moment $\left(7\left\langle T_{z}\right\rangle \mu_{B}\right)$ of the spin density distribution, can be determined from the integrals of the XMCD spectrum and the isotropic absorption spectrum ${ }^{2}$. Since the sum rule contains two terms, the contributions of the intrinsic spin moment and the magnetic dipole moment cannot be readily determined. The latter is known to be negligibly small for cubic symmetry only ${ }^{2}$. Limitations of the spin sum rule and the importance of the magnetic dipole term in noncubic environments such as surfaces and interfaces were studied with electronic band structure calculations for iron, cobalt and nickel ${ }^{4}$. The seperate determination of spin and magnetic dipole moments with an angle averaging spin sum rule was demonstrated theoretically ${ }^{5}$ and experimentally ${ }^{6}$ for a highly anisotropic $3 d$ transition metal system where the charge and spin densities were not spherically symmetric. The magnetic dipole moments of single cobalt atoms and nanoparticles (NPs) were quantified by combining the effective spin moment determined from measured XMCD spectra using the spin sum rule with the intrinsic spin moment calculated in the local spin density approximation ${ }^{7}$. According to ab initio studies, the $\left\langle T_{z}\right\rangle$ term, in general, cannot be neglected in the spin sum rule for systems with low dimensionalities like monolayers and monatomic wires ${ }^{8}$ and clusters ${ }^{9}$. Anisotropies of the spin density distributions causing large magnetic dipole moments were also investigated in molecules on surfaces like $\mathrm{Cu}$-phthalocyanine on $\mathrm{Ag}(001)^{10}$ and Fe-octaethylporphyrin on $\mathrm{Cu}(001)^{11}$.

For many examples, the measured contribution of the magnetic dipole moment can be regarded as a disadvantage of the XMCD impeding the extraction of the spin moment in a straight-forward way. In this work, we employ it as a monitor for changes in the electronic structure as it is caused by phase transitions. In order to show the potential of the XMCD technique, we chose magnetite $\left(\mathrm{Fe}_{3} \mathrm{O}_{4}\right)$ as an example because in magnetite the average Fe dipole moment in the low-temperature phase according to our electronic structure calculations is sufficiently large to be reliably quantified with XMCD. In addition, for magnetite XMCD is also site-selective giving the possibility to distinguish between Fe ions at different lattice sites.

Magnetite crystallizes in the inverse spinel structure in a cubic (isometric) crystal system, with the oxide anions arranged in a cubic close-packed lattice while the Fe cations are located on octahedral and tetrahedral lattice sites 
in between. In particular, $\mathrm{Fe}^{3+}$ occupies the tetrahedral sites (also denoted $\mathrm{A}$ sites in the literature) and half of the octahedral sites (B sites), whereas the $\mathrm{Fe}^{2+}$ ions are located at the remaining octahedral sites. For a schematic diagram of the monoclinic P2/c unit cell of magnetite containing 56 atoms we refer to Fig. 1 in Ref. [17]. The strongest interaction is the superexchange between $\mathrm{Fe}^{3+}$ on octahedral and $\mathrm{Fe}^{3+}$ on tetrahedral sites which is negative resulting in an antiparallel orientation of spins of different sublattices.

At low temperatures, magnetite undergoes a phase transition that was discovered in 1939 by Verwey as a rapid change of the electronic conductivity at a certain temperature and was named after him as the Verwey transition (VT). In the original article ${ }^{12}$ it was described as "a sudden increase of the resistance at approximately $117 \mathrm{~K}$ by a factor of the order 100 ". The resistance of a magnetite sample was compared with the one of a sample which was further oxidized and described as "samples containing an excess of $\mathrm{Fe}_{2} \mathrm{O}_{3}$ show a much smaller jump in the curve at about $120 \mathrm{~K}$ or even merely a change in the temperature coefficient" ${ }^{\prime 2}$. Many years later it was found by heat capacity studies ${ }^{13}$ that with increasing deviation $\delta$ in $\mathrm{Fe}_{3-\delta} \mathrm{O}_{4}$ from ideal magnetite stoichiometry the VT changes from first to second order and that the VT temperature decreases linearly with $\delta$.

The refined determination of the structural changes at the VT based on diffraction measurements ${ }^{14,15}$ initiated several theoretical investigations with modern methods ${ }^{16-18}$. The most significant structural change connected to the VT was explained in the work of H.-T. Jeng et al. ${ }^{17}$. They reported that in the low-temperature monoclinic unit cell, a charge and orbital ordering is related to one of the $\mathrm{Fe}^{3+}$ ions on an octahedral lattice site being pulled inwards the cube diagonal of the sub-unit cell yielding a lower energy of the system due to intersite Coulomb attraction with $t_{2 g}$ orbitals of three neighboring $\mathrm{Fe}^{2+}$ ions.

With resonant $\mathrm{x}$-ray scattering the changes in the structural, charge and orbital order were studied in dependence of the temperature across the VT using certain diffraction peaks to indicate structural, charge or orbital order ${ }^{19-21}$. The interpretation of the diffraction peaks was discussed and became a controversy.

In a structural study ${ }^{21}$ using high-resolution synchrotron $\mathrm{x}$-ray powder diffraction data of magnetite the charge disproportion found by J. P. Wright et al. ${ }^{15}$ was confirmed between the $\mathrm{B} 1$ and $\mathrm{B} 2$ sites but

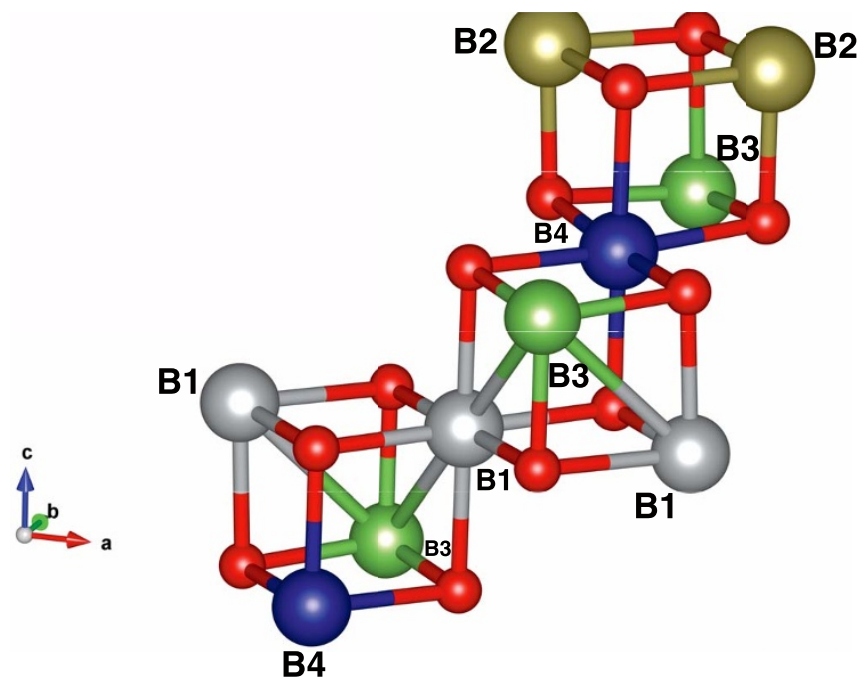

Figure $1 \mid$ Part of the magnetite unit cell in the monoclinic crystal structure. Oxygen atoms are drawn as small red spheres. Iron ions on different octahedral sites are drawn as large grey (B1), brown (B2), green (B3) and blue spheres (B4). The linear three-site structure B3-B1-B3 forms a trimeron with reduced $\mathrm{B} 1-\mathrm{B} 3$ bond lengths. The axes of the global coordinate system are also shown. not between the B3 and B4 sites (see Fig. 1). The picture of a wide distribution of different local environments around the octahedral Fe ions, caused by the condensation of several phonon modes, was favored over any bimodal charge disproportion on the octahedral sites $^{22}$.

Since diffraction studies of magnetite are hampered by different crystallite orientations and microtwinning, the full low-temperature superstructure has been quite recently determined with $\mathrm{x}$-ray diffraction from an almost single-domain grain of only $40 \mu \mathrm{m}$ size. There it has also been found that localized electrons are distributed over linear units of three Fe ions on octahedral sites which leads to the observed shortening of the two Fe-Fe distances. These three-Fe-units were viewed as quasiparticles and named "trimerons"23. More than 30 years earlier the fluctuations of what nowadays is called trimerons were observed above the VT temperature with critical diffuse neutron scattering ${ }^{24,25}$ and interpreted using the Yamada model based on electron-phonon coupling ${ }^{24}$ and later using a pseudospin-phonon theory ${ }^{25}$.

Below the VT temperature $t_{2 g}$ orbital order on octahedral $\mathrm{Fe}^{2+}$ sites has been directly found with resonant soft x-ray diffraction $(\mathrm{RSXD})^{26}$. For this method it is important to take into account that there is a layer at the surface of single crystals which contributes to the absorption but not to the diffraction of the soft $\mathrm{x}$-rays ${ }^{27}$. By studying the azimuthal dependence of the diffracted intensity it has been shown that the distortion of the $3 d$ orbitals towards monoclinic symmetry is by far larger than that of the lattice ${ }^{28}$. Ultrafast melting of the charge-orbital order leading to the formation of a transient phase, which had not been observed in equilibrium, was observed with time-resolved RSXD with a free-electron laser ${ }^{29}$. Very recently it was investigated on the ps time scale how trimerons become mobile across the $\mathrm{VT}^{30}$.

In magnetite NPs the VT has already been experimentally observed with different methods, e.g., magnetization measurements of a non-diluted system containing strong dipolar interparticle interactions $^{31}$ and magnetoresistance measurements of tunneling junctions of stacked monolayers of magnetite NPs of $5.5 \mathrm{~nm}$ average diameter for which a VT temperature of $96 \mathrm{~K}$ has been determined ${ }^{32}$. In these studies it has been found that the VT temperature significantly decreases when decreasing the size of the NPs to a small fraction $^{31}$ but that it is not very sensitive to small changes of the order of $10 \%$ of the particle size $e^{32}$.

However, one may note that in magnetometry, the VT can only hardly be distinguished from a spin reorientation transition that occurs in magnetite at slightly higher temperatures $\left(T_{S R} \approx\right.$ $132 \mathrm{~K}^{33}$ ). The spin reorientation is characterized by a vanishing magnetocrystalline anisotropy constant $K_{1}$, which is negative at room temperature. With decreasing temperature it decreases, acquiring a minimum at a temperature of about $250 \mathrm{~K}^{34}$. With further decreasing temperature, it increases and becomes positive at $T_{S R}$. Referring to the vanishing value of $K_{1}$, this temperature is also called isotropy point in literature. For single crystalline samples, the different transition temperatures were measured by recording the saturation isothermal remanent magnetization along a principal axis of the single crystal $^{33}$. For the case of an arbitrary orientation of the magnetic field with respect to the principal crystallographic axes, the effects of $T_{S R}$ and the VT on the remanence could not be clearly separated. For polycrystalline samples it seems to be impossible to distinguish between the two transitions. As pointed out by Rezníček et al. ${ }^{34}$, there exists a correlation between the spin reorientation and the VT. In this work, the authors connected the contribution to $K_{1}$ which leads to an anomalous increasing behavior with decreasing temperature between $250 \mathrm{~K}$ and $T_{S R}$ to a local charge and orbital ordering that becomes long-range and stable for temperatures below the VT.

The main purpose of the present paper is to study the magnetic moments, in particular the magnetic dipole moment of Fe as a function of temperature across the phase transitions in magnetite NPs 
with a diameter of $6 \mathrm{~nm}$ and a $200 \mathrm{~nm}$ thick polycrystalline magnetite film which serves as a reference. For this purpose a combination of experimental and theoretical methods, i.e. XANES, XMCD, vibrating sample magnetometry (VSM) and electronic structure calculations based on density functional theory, has been used. As it will be described in detail below, a transition of the dipole moment of the Fe $3 d$ spin-density distribution was experimentally found well above the VT and theoretically explained.

\section{Results}

Density functional theory. Calculations of the electronic structure were performed based on density functional theory on cubic (high temperature) and monoclinic (low temperature) structures of magnetite. Details of the calculations are described in the methods section. We have considered a monoclinic P2/c structure with 56 atoms per unit cell obtained by using high-resolution neutron and x-ray powder-diffraction data by Wright et al. ${ }^{14,15}$. A part of the monoclinic unit cell with its four inequivalent octahedral lattice sites, labelled B1-B4, is shown in Fig. 1. The resulting magnetic moments are shown in table I. The Fe sites are named as shown in Fig. 1 and like in Ref. [17]. Our calculated values of $\left\langle T_{z}\right\rangle$ for the cubic structure are negligible $\left(\sim 10^{-5} \mu_{B}\right)$ whereas for the monoclinic structure, they are quite large, especially for the octahedral B sites. It is clearly observed from the calculations that the $\mathrm{Fe}^{2+}$ ion has a larger contribution than $\mathrm{Fe}^{3+}$ (see Table I, where we compile the calculated values of $\left.7\left\langle T_{z}\right\rangle\right)$. At the $\mathrm{B} 4$ site, the contribution is as large as $-1.44 \mu_{B}$ with an anti-parallel alignment to the spin moment. It is only partly compensated by the second largest contribution of $0.72 \mu_{B}$ at the B1 site with a parallel alignment to the spin moment. Therefore, these octahedral sites substantially modify the effective spin moments, as detected in the XMCD measurements.

We also calculated the Fe orbital magnetic moments for the monoclinic phase in the $a, b$ and $c$ directions as defined in Fig. 1. The sizeable orbital moments can be as large as $0.035 \mu_{B}$ along the $c$ direction for Fe ions on $\mathrm{B} 4$ sites and $0.031 \mu_{B}$ along the $b$ direction for Fe ions on $\mathrm{B} 1$ sites. For all other sites, the orbital moments are almost isotropic with absolute values of $0.015 \mu_{B}$ to $0.019 \mu_{B}$. The signs of the orbital moments follow the spin moments. To further analyze the large values of $\left\langle T_{z}\right\rangle$, we show in Fig. 2, the $\mathrm{m}_{l}$ projected density of states (DOS) for different octahedral sites of the monoclinic phase. The reason for the high $\left\langle T_{z}\right\rangle$ value is the full occupancy of the $\mathrm{d}_{x^{2}-y^{2}}$ orbital in the spin down channel of the $\mathrm{Fe}^{2+}$ ion on the $\mathrm{B} 4$ site. For the $\mathrm{Fe}^{3+}$ ion, this orbital is unoccupied. Similarly, a high value (half of that of the B4 site, and with parallel alignment to the spin-moment) is observed for the $\mathrm{Fe}^{2+}$ ion on the $\mathrm{B} 1$ site. The large value also for this site is coupled to the fact that the $d_{x z}$ and $d_{y z}$ orbitals are occupied, although only partially. Again, for the $\mathrm{Fe}^{3+}$ ion, both these orbitals are unoccupied, and do not contribute to $\left\langle T_{z}\right\rangle$.

All of our calculated results can be understood from the $\left\langle T_{z}\right\rangle$ matrix elements explicitly provided in the paper by Crocombette et al. ${ }^{35}$. For $\mathrm{Fe}^{3+}$, the spin-up channel is completely filled with almost

Table I | Charge, spin and magnetic dipole moments for 3 d orbitals of Fe atoms at different sites in the monoclinic unit cell. Also, effective moments $\left(\mu_{S \text {, eff }}=-2\left\langle S_{z}\right\rangle \mu_{B}+7\left\langle T_{z}\right\rangle \mu_{B}\right)$ are provided. The Fe sites are named as in Ref. [17]

\begin{tabular}{lcccc} 
Fe site & d-charge & $-2\left\langle S_{z}\right\rangle$ & $7\left\langle T_{z}\right\rangle$ & $\mu_{S, \text { eff }}\left(\mu_{B}\right)$ \\
\hline A1 & 5.91 & -3.98 & -0.015 & -3.995 \\
A2 & 5.91 & -3.98 & 0.025 & -3.955 \\
B1 & 6.08 & 3.67 & 0.72 & 4.39 \\
B2 & 5.82 & 4.14 & 0.043 & 4.183 \\
B3 & 5.85 & 4.08 & 0.027 & 4.107 \\
B4 & 6.1 & 3.64 & -1.44 & 2.20 \\
\hline
\end{tabular}

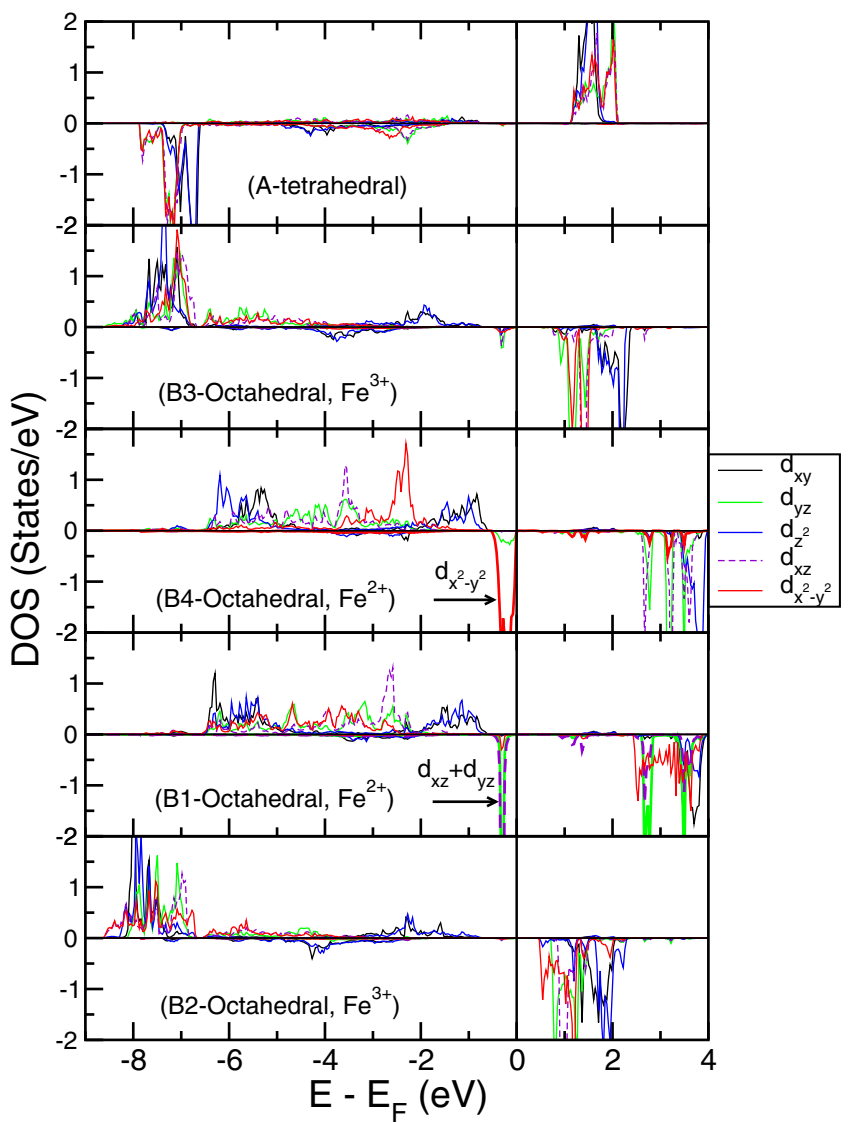

Figure $2 \mid$ Spin-resolved $\mathrm{m}_{l}$ projected density of states for the monoclinic structure. DOS at the tetrahedral A site along with four different octahedral B sites are shown. The Fe sites are named as shown in Fig. 1 and like in Ref. [17]. The specific orbitals responsible for the large contribution in the $\left\langle T_{z}\right\rangle$ part are indicated by arrows. The energies in the $\mathrm{x}$-axes are plotted with respect to the Fermi level $\mathrm{E}_{F}$.

no occupancy in the spin-down channel and therefore, much smaller $\left\langle T_{z}\right\rangle$ contributions are observed. Note that XANES and XMCD are sensitive to the unoccupied DOS so that in these experiments the magnetic moments due to the lack of unoccupied states instead of the existence of occupied states is probed. Also note that even for $\mathrm{Fe}^{3+}$ in the monoclinic phase due to the low symmetry structure compared to the cubic one, we have higher contribution to $\left\langle T_{z}\right\rangle$ compared to the cubic one. Obviously, there is no noticeable distinction between $\mathrm{Fe}^{2+}$ and $\mathrm{Fe}^{3+}$ in the cubic phase as the charge ordering is non-existent in the metallic phase.

X-ray absorption spectroscopy. The XANES and XMCD spectra are defined as the sum and difference of two spectra obtained with opposite helicities of the incident circularly polarized soft x-ray radiation, respectively. Measured XANES spectra and corresponding XMCD spectra of the NPs and the film sample are shown in Fig. 3 and Fig. 4, respectively, at the $\mathrm{Fe} L_{2,3}$ absorption edges for various temperatures. The temperature and magnetic field histories of both samples were identical, i.e. both samples were field-cooled to $4 \mathrm{~K}$ in a magnetic field of $0.5 \mathrm{~T}$ and subsequently heated stepwise to and measured at the temperatures indicated by the data points in Fig. 5 in a magnetic field of $3 \mathrm{~T}$. The NPs offer significant changes of the XANES intensity and the XMCD intensity between $50 \mathrm{~K}$ and $100 \mathrm{~K}$ and the film sample between $150 \mathrm{~K}$ and $175 \mathrm{~K}$. For both samples the spectra are devided into two groups: Spectra for temperatures below the change are shown as blue lines, and above as red lines.

XMCD has the advantage that spin and orbital moments can be determined element-specifically by use of sum rules ${ }^{1,2}$. For the deter- 


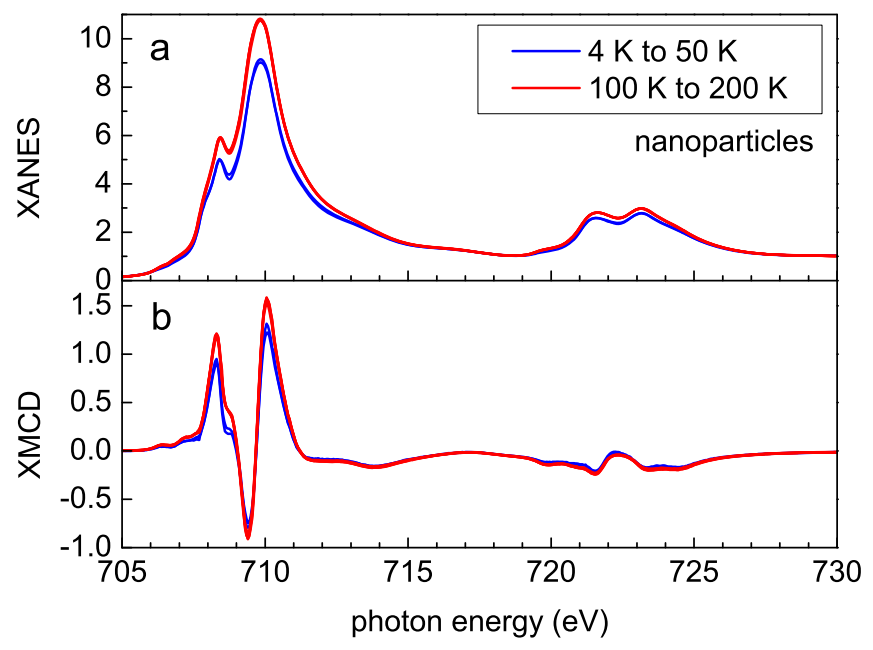

Figure $3 \mid$ Measured XANES (a) and corresponding XMCD spectra (b) of the nanoparticles at the $L_{2,3}$ absorption edges. The XANES spectra are shown after subtracting a linear background and normalizing at a photon energy in the post-edge region.

mination of the magnetic moments via sum rules, we normalized the spectra in the usual way, i.e. first subtracted a constant background, then normalized at a photon energy above the absorption edge and finally subtracted a step like function. Please note that the spectra have been measured from $680 \mathrm{eV}$ to $780 \mathrm{eV}$ in order to be able to perform a reliable normalization. In Fig. 3 and Fig. 4 only the physically relevant spectral range from $705 \mathrm{eV}$ to $730 \mathrm{eV}$ has been shown for the sake of clarity. The effective spin moment $\left(\mu_{S, \text { eff }}=-2\left\langle S_{z}\right\rangle \mu_{B}\right.$ $\left.+7\left\langle T_{z}\right\rangle \mu_{B}\right)$ is defined as the intrinsic spin moment $\left(-2\left\langle S_{z}\right\rangle \mu_{B}\right)$ plus the magnetic dipole moment $\left(7\left\langle T_{z}\right\rangle \mu_{B}\right)$ of the spin-density distribution $^{2}$ where $z$ is defined by the magnetization direction. Its normalized values determined from the measured XMCD spectra as a function of temperature are shown for the NPs in Fig. 5a and for the film in Fig. 5b. The effective Fe spin moment of the NPs increases between $50 \mathrm{~K}$ and $100 \mathrm{~K}$ by about $7 \%$ relative to the value at $100 \mathrm{~K}$ and for the film between $150 \mathrm{~K}$ and $175 \mathrm{~K}$ by $16 \%$ relative to the value at $175 \mathrm{~K}$. The magnetic moments of the NPs and the film sample were also measured with VSM and are shown for comparison as gray lines in Fig. 5a and Fig. 5b, respectively. Increases of the

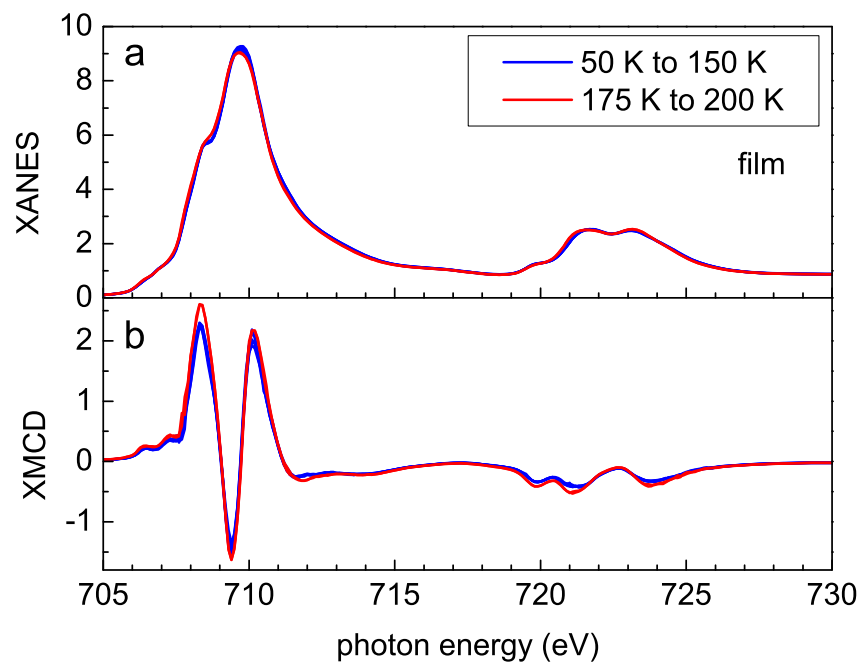

Figure $4 \mid$ Measured XANES (a) and corresponding XMCD spectra (b) of the film sample at the $L_{2,3}$ absorption edges. The XANES spectra are shown after subtracting a linear background and normalizing at a photon energy in the post-edge region.

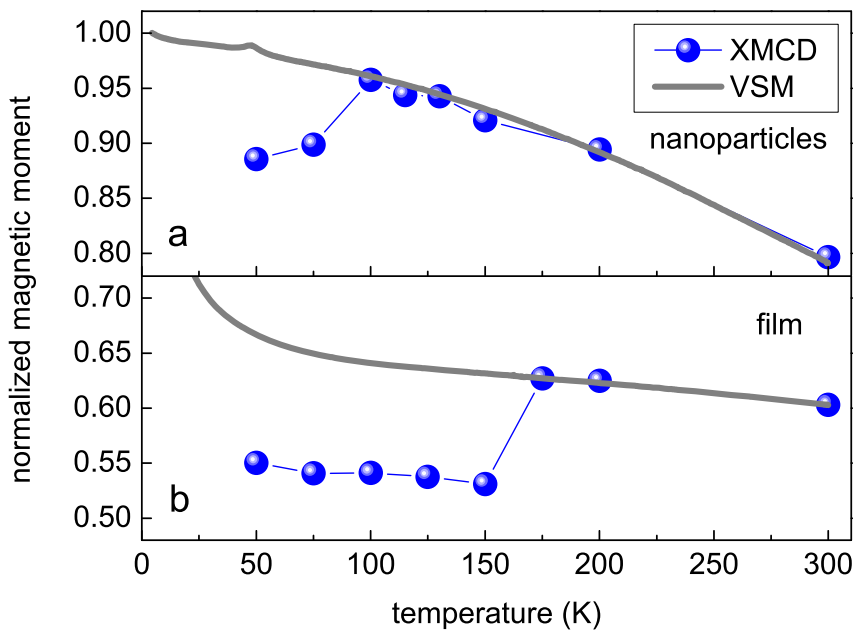

Figure 5 Normalized effective Fe spin moments as determined from the measured XMCD spectra (solid symbols) and the magnetic moment measured with VSM (thick gray line) as a function of temperature for the magnetite NPs (a) and the film sample (b). Errors due to the reproducibility of the evaluation of the XMCD spectra are within the symbol size.

magnetic moments with increasing temperatures were not observed with VSM. This important result is due to the fact that the magnetic dipole moment is observable with XMCD but not with VSM as discussed in the following section.

\section{Discussion}

The electronic structure calculations show a strong change of the magnetic dipole moment in magnetite when the structure of the lattice changed from cubic to monoclinic. These changes are most significant for the case of $\mathrm{Fe}^{2+}$ ions on octahedral lattice sites (B1, B4) which is in agreement to the experimental XMCD data. The transition observed with XMCD (Fig. $3 \mathrm{~b}$ and Fig. 4b) is accompanied by a change of the asymmetry, which is defined as the XMCD spectrum devided by the XANES spectrum after adequate background subtraction and normalization, at the photon energies $708.2 \mathrm{eV}$ and $710 \mathrm{eV}$ which mainly correspond to octahedral sites ${ }^{38}$. The transition is also observed in the effective Fe spin moment (Fig. 5). However, in general this transition could be assigned to a change in the spin moment, the magnetic dipole moment or both at the same time. To analyse which contribution to the effective spin moment is mainly responsible for its significant change, the XMCD results are compared to magnetometry data obtained with VSM. The $\left\langle T_{z}\right\rangle$ term only shows up in XMCD measurements because they involve an excitation from a core level to an unoccupied valence level. It does not show up in magnetometry or other probes that detect the macroscopic magnetization. For further discussion, see Ref. [39] and Ref. [40]. As shown in Fig. 5, there is no corresponding increase of the total magnetic moment in the VSM data although this method certainly is sufficiently sensitive to detect a change of about $7 \%$ for the NPs and $16 \%$ for the film. Therefore the transition observed with XMCD cannot be due to a change in the intrinsic Fe spin moment but must be due to a change in the second term of the sum rule for the effective spin moment ${ }^{2}$, i.e. the $\left\langle T_{z}\right\rangle$ term which describes the dipole moment of the Fe spin-density distribution. This conclusion is in accordance with our theoretical treatment of the VT.

In Fig. 6, we compare the experimentally determined values of the effective spin magnetic moment of the NPs with the theoretically observed ones. The calculated $0 \mathrm{~K}$ effective moments, averaged over all Fe sites have been multiplied with the Bloch law $1-0.96$ $(T / 850 \mathrm{~K})^{3 / 2}$ to obtain magnetic moments as a function of temperature. The theoretical moments are seen to drop at the known VT 
temperature for the bulk, where the crystal structure changes from cubic to monoclinic. Again, it is the value of $\left\langle T_{z}\right\rangle$ that changes. The relative change of the moments nicely agrees between theory and experiment in particular for the NPs although $\left\langle T_{z}\right\rangle$ was calculated along one crystallographic direction (the $c$ direction as indicated in Fig. 1) whereas in the measurement it was averaged over all directions due to the random orientation of the NPs. Nevertheless, it is clear that a detailed measurement of $\left\langle T_{z}\right\rangle$ shows up in an unprecedented way. This then becomes a valuable characterization tool, that signals not only changes in the local geometry but also the local electronic structure. It is likely that in general, detailed knowledge of $\left\langle T_{z}\right\rangle$ is a valuable tool for understanding local changes in the crystal- and electronic structure, not only for complex oxides but also complex actinide and rare-earth compounds.

For $3 d$ transition metals it has been pointed out by Stöhr and König ${ }^{5}$ that spin-orbit correction terms in the perturbative calculation of $\left\langle T_{\alpha}\right\rangle$ can be neglected to a good approximation, leading to the numerical relation $\left\langle T_{x}\right\rangle+\left\langle T_{y}\right\rangle+\left\langle T_{z}\right\rangle \approx 0$ and to the possibility to average out the magnetic dipole moment by magnetizing and measuring the sample along the three cartesian axes $(\alpha=x, y, z)$. In the case of our magnetite NPs the average magnetic dipole moment would vanish in each single measurement geometry because the NPs are randomly oriented like in a powder sample so that the integration of the $\mathrm{z}$ component $T_{z}=S_{z}\left(1-3 \cos ^{2} \theta\right) / 2^{4}$ over the solid angle yields zero. However, according to Ederer et al. the effects of spin-orbit coupling are larger for low-dimensional systems ${ }^{8}$ and in particular the relation $\left\langle T_{x}\right\rangle+\left\langle T_{y}\right\rangle+\left\langle T_{z}\right\rangle \approx 0$ is strongly violated for monoatomic $\mathrm{Fe}, \mathrm{Co}$ and $\mathrm{Ni}$ wires ${ }^{41}$. In addition, spin-orbit coupling is larger for $3 d$ oxides like magnetite than for $3 d$ metals due to a stronger localization of the $3 d$ electrons. The large spin-orbit coupling becomes noticeable by the large magnetic anisotropy constant in the monoclinic phase of magnetite ${ }^{34}$. Moreover, it causes that the $3 d$ charge distribution is no longer independent from the spin direction $^{5}$. Therefore the effect of spin-orbit coupling in the determination of the components $\left\langle T_{\alpha}\right\rangle$ can no longer be neglected, i.e. the terms containing the spin-flip operators do no longer vanish (see Appendix $B$ in Ref. [40]). For the experimental determination of the magnetic dipole moment this means that at least a part of the magnetic dipole moment is fixed to the magnetization direction but not to the crystal lattice when the crystal (here each NP) is rotated relative to the magnetic field. In this case it is not possible to separate the intrinsic spin moment and the magnetic dipole moment solely by angle dependent XMCD measurements because the precondition for averaging out the magnetic dipole moment is no longer fulfilled. The present results for magnetite NPs with $6 \mathrm{~nm}$ diameter demonstrate

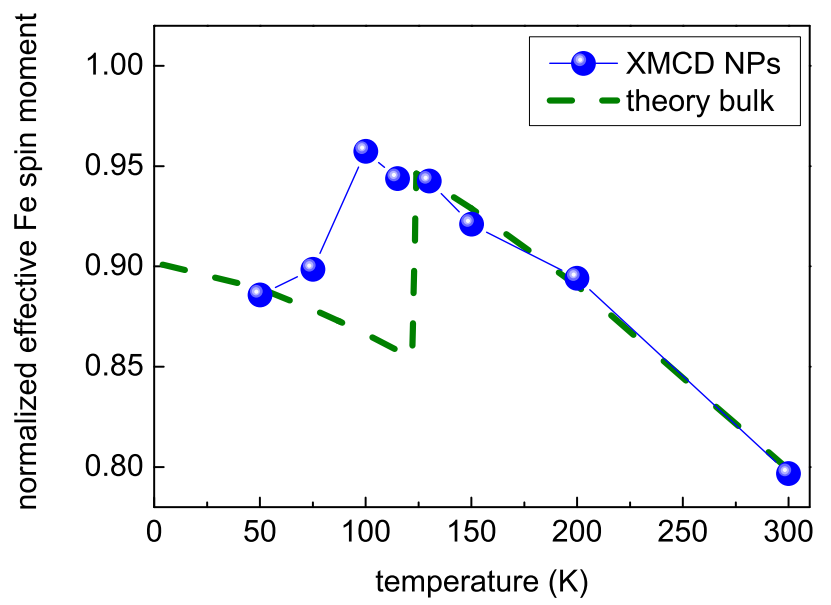

Figure 6 | Normalized experimental effective Fe spin magnetic moments of magnetite NPs (blue symbols) compared to theoretically obtained results (dashed line). that the magnetic dipole moment indeed is not averaged out in the monoclinic low-temperature phase.

The small average Fe orbital moments of magnetite with values below $0.03 \pm 0.02 \mu_{B}$ as determined with XMCD experiments in Ref. [37] do not exclude sizeable local spin-orbit effects because, according to our calculations, the absolute values of the Fe orbital moments vary strongly with the lattice site and their signs follow the spin moments so that they partly cancel each other like the spin moments and magnetic dipole moments do. In addition, the NPs will show higher values of the Fe orbital moments due to surface effects. For the $200 \mathrm{~nm}$ thick polycrystalline magnetite film which served as a bulk reference the VT was observed with VSM at $120 \mathrm{~K}$ as shown in the Supplementary Information. With field-cooled/zero-field-cooled measurements peaks in the blocking temperature distributions were found at $120 \mathrm{~K}$, which indicate the VT because their temperature positions were independent of the magnitude of small magnetic fields. Interestingly, at this temperature no transition was observed with XMCD in accordance with the results in Ref. [37]. Surprisingly, with XMCD a transition was detected well above the VT temperature, i.e. between $150 \mathrm{~K}$ and $175 \mathrm{~K}$. An error in the temperature measurement can be excluded because with the used experimental setup, i.e. the high-field endstation at beamline UE46-PGM1 at the electron storage ring BESSY II, various transitions between $4 \mathrm{~K}$ and $300 \mathrm{~K}$ have been found at the known transition temperatures. Therefore we believe that the enhanced absolute values of the magnetic dipole moments of the monoclinic structure persist above the VT temperature until a temperature between $150 \mathrm{~K}$ and $175 \mathrm{~K}$. This is in agreement with the unusual temperature dependence of the magnetocrystalline anisotropy that can be explained by a local charge and orbital ordering well above the VT as suggested by Rezníček et $\mathrm{al}^{34}$. In this sense, our work gives the first experimental evidence for this interpretation of changes in the electronic structure above the VT.

For the NPs, the spectral shapes in Fig. 3a and Fig. 3b indicate that a charge transfer could be present to ligands which have not been washed out completely after the synthesis ${ }^{38}$, or that the NPs are further oxidized at the surface ${ }^{42}$. According to our experience both processes, charge transfer to ligands and oxidation at the surface, result in similar spectra ${ }^{38}$. However, the transition observed with XMCD disppeared after storing the NPs 17 months in solution. The transition was re-observed with XMCD after treating the NPs in-situ with a Hydrogen plasma which resulted in perfect magnetite composition according to the XANES and XMCD spectra. Therefore we confidently associate the transition observed with XMCD with magnetite.

Compared to the film sample, the transition temperature of the NPs is reduced. A reduction of the VT temperature of NPs is known from other experiments with different methods ${ }^{26,27}$ and was explained with spin canting and reduced thermal stability at the surface $^{43}$. In our NPs, spin canting has also been experimentally observed as a finite slope at high magnetic fields in magnetization hysteresis measurements with $\mathrm{XMCD}^{44}$ and with Mößbauer spectroscopy as described in Ref. [45]. Thus, a reduced VT temperature seems to be reasonable and can explain the reduced transition temperature measured with XMCD. For the case of NPs, magnetometry data may not be sufficient to detect changes in the magnetic properties caused by a phase transition, due to a strong influence of blocking effects as discussed in the Supplementary Information where we present VSM data of our NPs. This shows the importance of measurements of the magnetic dipole moment as a sensitive detector for local changes of the electronic structure.

One might ask whether it is reasonable to attribute the observed transition of the magnetic dipole moment to quasiparticles called trimerons. Trimerons consist of a linear unit of three Fe ions on octrahedral sites in which a $t_{2 g}$ minority-spin electron from the central $\mathrm{Fe}^{2+}$ donor site is distributed over two adjecent $\mathrm{Fe}^{3+}$ acceptor 
sites $^{23}$. This distribution tends to shorten the two corresponding $\mathrm{Fe}^{2+}-\mathrm{Fe}^{3+}$ distances which was recently observed with $\mathrm{x}$-ray scattering $^{23}$. Intuitively, one might expect that this donated $t_{2 g}$ minorityspin electron creates an anisotropy of the spin density distribution which is accompanied by a magnetic dipole moment. According to our electronic structure calculations the B1-B3 bond length is shorter than other bond lenghts in the unit cell which corresponds to B3-B1-B3 trimerons as shown in Fig. 1. The first potential donor site, $\mathrm{B} 1 \mathrm{Fe}^{2+}$, is part of a trimeron and its $\mathrm{d}_{x z}$ and $\mathrm{d}_{y z}$ orbitals exhibit a magnetic dipole moment of $0.72 \mu_{B}$. Contrary, the second potential donor site, $\mathrm{B} 4 \mathrm{Fe}^{2+}$, which carries the largest magnetic dipole moment of $-1.44 \mu_{B}$ in its $\mathrm{d}_{x^{2}-y^{2}}$ orbital, is not part of a trimeron. Therefore it is not sufficient to assign the observed total magnetic dipole moment just to trimerons.

Summary. In summary, magnetite NPs with $6 \mathrm{~nm}$ diameter and a $200 \mathrm{~nm}$ thick polycrystalline magnetite film which served as a bulk reference were experimentally investigated with XANES, XMCD and VSM and compared with electronic structure calculations based on density functional theory. The measured spectra of the NPs offered significant changes in the white line intensity and the $\mathrm{XMCD}$ asymmetry between $50 \mathrm{~K}$ and $100 \mathrm{~K}$. The film sample also showed the transition in the XMCD asymmetry which appeared between $150 \mathrm{~K}$ and $175 \mathrm{~K}$. A sum rule analysis of the XMCD spectra revealed that the transition observed with XMCD is due to an increase of the effective $\mathrm{Fe}$ spin moment with increasing temperature which was attributed to a change of the dipole moment of the Fe $3 d$ spin-density distribution. This conclusion was verified and explained with electronic structure calculations based on density functional theory. The large absolute value of the negative magnetic dipole moment in the monoclinic structure is caused by the full occupancy of the $\mathrm{d}_{x^{2}-y^{2}}$ orbital in the spin down channel of the $\mathrm{Fe}^{2+}$ ion on the $\mathrm{B} 4$ site resulting in a negative magnetic dipole moment, which is only partly compensated by the positive magnetic dipole moment of partially occupied $\mathrm{d}_{x z}$ and $\mathrm{d}_{y z}$ orbitals of the $\mathrm{Fe}^{2+}$ ion on the $\mathrm{B} 1$ site. Since the $\mathrm{B} 1$ site but not the B4 site is part of a trimeron it is not meaningful to attribute the observed total magnetic dipole moment just to trimerons. In the case of magnetite, the experimental results evidence the occurence of local charge and orbital ordering well above the VT as suggested in a recent publication $^{34}$. In general, detailed knowledge of the magnetic dipole moment is a valuable tool for understanding local changes in the crystal- and electronic structure, not only for complex oxides but also complex actinide and rare-earth compounds.

\section{Methods}

Synthesis of nanoparticles. The NPs were prepared using a one-pot water-in-oil microemulsion technique as described in detail elsewhere ${ }^{45} . \mathrm{FeCl}_{2}$ and $\mathrm{FeCl}_{3}$ were used as precursors, IGEPAL ${ }^{\circledR} \mathrm{CO}-520$ (polyoxyethylene (5) nonylphenylether) as stabilizing organic surfactant and ammonium hydroxide as catalyst. The water droplets coated by the surfactant act as nanoreactors in which the magnetite NPs are formed. The diameters of the NPs $(6.3 \pm 0.9 \mathrm{~nm})$ were determined with transmission electron microscopy averaging over a few hundred NPs. The NPs were prepared at the University of Duisburg-Essen, transported in liquid solution to Berlin, deposited onto substrates and transferred into ultra-high vacuum for the measurements.

X-ray absorption. The XANES and XMCD measurements were performed in the highfield endstation at beamline UE46-PGM1 with polarized synchrotron radiation from an elliptical undulator in the electron storage ring BESSY II of the HelmholtzZentrum Berlin. The total electron yield (TEY) method used in the present measurements to monitor the absorption of the soft $\mathrm{x}$-rays has an information depth which is determined by the mean free path of the secondary photoelectrons of $1 \mathrm{~nm}$ to $2 \mathrm{~nm}$. Since the magnetite NPs have a mean radius of $3 \mathrm{~nm}$, the element-specific XANES and XMCD spectra represent the average over the whole particle with a pronounced signal from the surface with respect to the core.

Magnetometry. The VSM measurements were performed with a Physical Properties Measurement System from the company Quantum Design which has a sensitivity of below $10^{-9} \mathrm{~J} / \mathrm{T}$ and is operated in the laboratory cluster at the Helmholtz-Zentrum Berlin.
Density functional theory. The generalized gradient approximation (GGA) as given by Perdew, Burke and Ernzerhof ${ }^{36}$ plus on-site Coulomb interaction $U$ to include strong electron correlation in the $d$-orbitals of Fe were used. The Coulomb parameter $U=4.5 \mathrm{eV}^{46}$ and the exchange parameter $J=0.89 \mathrm{eV}^{47}$ were used for all Fe- $d$ orbitals. The projector augmented wave method ${ }^{48,49}$ as implemented in plane-wave based density functional code $\operatorname{VASP}^{50}$ has been used for the calculations. The plane wave cutoff energy was set at $400 \mathrm{eV}$ energy. $12 \times 12 \times 12$ and $12 \times 12 \times 4$ MonkhorstPack k-point grids in the Brillouin zone were used for cubic and monoclinic structures respectively. The geometries were optimized until the force on all each atom was reduced to $0.1 \mathrm{eV} / \mathrm{nm}$.

1. Thole, B. T., Carra, P., Sette, F. \& van der Laan, G. X-ray circular dichroism as a probe of orbital magnetization. Phys. Rev. Lett. 68, 1943-1946 (1992).

2. Carra, P., Thole, B. T., Altarelli, M. \& Wang, X. X-ray circular dichroism and local magnetic fields. Phys. Rev. Lett. 70, 694-697 (1993).

3. Chen, C. T. et al. Experimental confirmation of the x-ray magnetic circular dichroism sum rules for iron and cobalt. Phys. Rev. Lett. 75, 152 (1995).

4. Wu, R. \& Freeman, A. J. Limitation of the magnetic-circular-dichroism spin sum rule for transition metals and importance of the magnetic dipole term. Phys. Rev. Lett. 73, 1994 (1994).

5. Stöhr, J. \& König, H. Determination of spin- and orbital-moment anisotropies in transition metals by angle-dependent x-ray magnetic circular dichroism. Phys. Rev. Lett. 75, 3748 (1995).

6. Weller, D. et al. Microscopic origin of magnetic anisotropy in $\mathrm{Au} / \mathrm{Co} / \mathrm{Au}$ probed with x-ray magnetic circular dichroism. Phys. Rev. Lett. 75, 3752 (1995).

7. Gambardella, P. et al. Giant magnetic anisotropy of single cobalt atoms and nanoparticles. Science 300, 1130-1133 (2003).

8. Ederer, C., Komelj, M. \& Fähnle, M. Magnetism in systems with various dimensionalities: A comparison between Fe and Co. Phys. Rev. B 68, 052402 (2003).

9. Sipr, O., Minar, J. \& Ebert, H. On the importance of the magnetic dipole term $\mathrm{T}_{z}$ in analyzing X-ray magnetic circular dichroism spectra of clusters. Europhys. Lett. 87, 67007 (2009).

10. Stepanow, S. et al. Giant spin and orbital moment anisotropies of a Cuphthalocyanine monolayer. Phys. Rev. B 82, 014405 (2010).

11. Herper, H. C. et al. Iron porphyrin molecules on $\mathrm{Cu}(001)$ : Influence of adlayers and ligands on the magnetic properties. Phys. Rev. B 87, 174425 (2013).

12. Verwey, E. J. W. Electronic conduction of magnetite $\left(\mathrm{Fe}_{3} \mathrm{O}_{4}\right)$ and its transition point at low temperatures. Nature 3642, 327-328 (1939).

13. Shepherd, J. P., Koenitzer, J. W., Aragon, R., Spalek, J. \& Honig, J. M. Heat capacity and entropy of nonstoichiometric magnetite $\mathrm{Fe}_{3(1-\delta)} \mathrm{O}_{4}$ : The thermodynamic nature of the Verwey transition. Phys. Rev. B 43, 8461-8471 (1991).

14. Wright, J. P., Attfield, J. P. \& Radaelli, P. G. Long range charge ordering in magnetite below the Verwey transition. Phys. Rev. Lett. 87, 266401 (2001).

15. Wright, J. P., Attfield, J. P. \& Radaelli, P. G. Charge ordered structure of magnetite $\mathrm{Fe}_{3} \mathrm{O}_{4}$ below the Verwey transition. Phys. Rev. B 66, 214422 (2002).

16. Leonov, I., Yaresko, A. N., Antonov, V. N., Korotin, M. A. \& Anisimov, V. I. Charge and orbital order in $\mathrm{Fe}_{3} \mathrm{O}_{4}$. Phys. Rev. Lett. 93, 146404 (2004).

17. Jeng, H.-T., Guo, G. Y. \& Huang, D. J. Charge-orbital ordering and Verwey transition in magnetite. Phys. Rev. Lett. 93, 156403 (2004).

18. Piekarz, P., Parlinski, K. \& Oleś, A. M. Mechanism of the Verwey transition in magnetite. Phys. Rev. Lett. 97, 156402 (2006).

19. Lorenzo, J. E. et al. Charge and orbital correlations at and above the Verwey phase transition in magnetite. Phys. Rev. Lett. 101, 226401 (2008).

20. Garcia, J. et al. Reexamination of the temperature dependence of resonant reflections in highly stoichiometric magnetite. Phys. Rev. Lett. 102, 176405 (2009).

21. Blasco, J., Garcia, J. \& Subias, G. Structural transformation in magnetite below the Verwey transition. Phys. Rev. B 83, 104105 (2011).

22. Subias, G. et al. Structural distortion, charge modulation and local anisotropies in magnetite below the Verwey transition using resonant $\mathrm{x}$-ray scattering. J. Synchrotron Rad. 19, 159-173 (2012).

23. Senn, M. S., Wright, J. P. \& Attfield, J. P. Charge order and three-site distortions in the Verwey structure of magnetite. Nature 481, 173-176 (2012).

24. Fujii, Y., Shirane, G. \& Yamada, Y. Study of the $123-\mathrm{K}$ phase transition of magnetite by critical neutron scattering. Phys. Rev. B 11, 2036 (1975).

25. Shapiro, S. M., Iizumi, M. \& Shirane, G. Neutron scattering study of the diffuse critical scattering with the Verwey transition in magnetite $\left(\mathrm{Fe}_{3} \mathrm{O}_{4}\right)$. Phys. Rev. B 14, 200-207 (1976)

26. Schlappa, J. et al. Direct observation of $t_{2 g}$ orbital ordering in magnetite. Phys. Rev. Lett. 100, 026406 (2008).

27. Chang, C. F. et al. Intrinsic and extrinsic $\mathrm{x}$-ray absorption effects in soft $\mathrm{x}$-ray diffraction from the superstructure in magnetite. Phys. Rev. B 83, 073105 (2011).

28. Tanaka, A. et al. Symmetry of orbital order in $\mathrm{Fe}_{3} \mathrm{O}_{4}$ studied by $\mathrm{Fe} L_{2,3}$ resonant Xray diffration. Phys. Rev. Lett. 108, 227203 (2012).

29. Pontius, N. et al. Time-resolved resonant soft $\mathrm{x}$-ray diffration with free electron lasers: Femtosecond dynamics across the Verwey transition in magnetite. Appl. Phys. Lett. 98, 182504 (2011).

30. de Jong, S. et al. Speed limit of the insulator-metal transition in magnetite. Nature Materials 3718 (2013). 
31. Goya, G. F., Berquo, T. S., Fonseca, F. C. \& Morales, M. P. Static and dynamic magnetic properties of spherical magnetite nanoparticles. J. Appl. Phys. 94, 3520-3528 (2003).

32. Poddar, P., Fried, T. \& Markovich, G. First-order metal-insulator transition and spin-polarized tunneling in $\mathrm{Fe}_{3} \mathrm{O}_{4}$ nanocrystals. Phys. Rev. B 65, 172405 (2002).

33. Özdemir, Ö. \& Dunlop, D. J. Low-temperature properties of a single crystal of magnetite oriented along principal magnetic axes. Earth and Planetary Science Letters 165, 229239 (1999).

34. Řezníček, R., Chlan, R., Štěpánková, H., Novák, P. \& Maryško, M. Magnetocrystalline anisotropy of magnetite. J. Phys.: Condens. Matter 24, 055501 (2012).

35. Crocombette, J. P., Thole, B. T. \& Jollet, F. The importance of the magnetic dipole term in magneto-circular $\mathrm{x}$-ray absorption dichroism for $3 \mathrm{~d}$ transition metal compounds. J. Phys.: Condens. Matter 8, 4095 (1996).

36. Perdew, J. P., Burke, K. \& Ernzerhof, M. Generalized gradient approximation made simple. Phys. Rev. Lett. 77, 3865-3868 (1996).

37. Goering, E. J., Lafkioti, M., Gold, S. \& Schütz, G. Absorption spectroscopy and $\mathrm{XMCD}$ at the Verwey transition of $\mathrm{Fe}_{3} \mathrm{O}_{4}$. J. Magn. Magn. Mater. 310, e249-e251 (2007).

38. Warland, A. et al. Effect of silica capping on the oxidation of $\mathrm{Fe}_{3} \mathrm{O}_{4}$ nanoparticles in dispersion revealed by x-ray absorption spectroscopy. Phys. Rev. B 85, 235113 (2012)

39. van der Laan, G. Angular momentum sum rules for x-ray absorption. Phys. Rev. B 57, 112-115 (1998).

40. Stöhr, J. Exploring the microscopic origin of magnetic anisotropies with X-ray magnetic circular dichroism (XMCD) spectroscopy. J. Magn. Magn. Mater. 200, 470-497 (1999).

41. Ederer, C., Komelj, M., Davenport, J. W. \& Fähnle, M. Comment on the analysis of angle-dependent X-ray magnetic circular dichroism in systems with reduced dimensionality. J. Electron Spectrosc. Relat. Phenom. 130, 97-100 (2003).

42. Pellegrin, E. et al. Characterization of nanocrystalline $\gamma-\mathrm{Fe}_{2} \mathrm{O}_{3}$ with synchrotron radiation techniques. Phys. Stat. Sol. (b) 215, 797-801 (1999).

43. Batlle, X. \& Labarta, A. Finite-size effects in fine particles: magnetic and transport properties. J. Phys. D: Appl. Phys. 35, R15-R42 (2002).

44. Warland, A. Röntgenabsorptionsspektroskopie an Fe-Oxid-Nanopartikeln Doctoral thesis, University Duisburg-Essen (2013).

45. Darbandi, M. et al. Nanoscale size effect on surface spin canting in iron oxide nanoparticles synthesized by the microemulsion method. J. Phys. D: Appl. Phys. 45, 195001 (2012).

46. Anisimov, V. I., Elfimov, I. S., Hamada, N. \& Terakura, K. Charge-ordered insulating state of $\mathrm{Fe}_{3} \mathrm{O}_{4}$ from first-principles electronic structure calculations. Phys. Rev. B 54, 4387-4390 (1996).

47. Anisimov, V. I., Zaanen, J. \& Andersen, O. K. Band theory and Mott insulators: Hubbard U instead of Stoner I. Phys. Rev. B 44, 943-954 (1991).
48. Blöchl, P. E. Projector augmented-wave method. Phys. Rev. B 50, 17953-17979 (1994).

49. Kresse, G. \& Joubert, D. From ultrasoft pseudopotentials to the projector augmented-wave method. Phys. Rev. B 59, 1758 (1999).

50. Kresse, G. \& Furthmüller, J. Efficient iterative schemes for $a b$ initio total-energy calculations using a plane-wave basis set. Phys. Rev. B 54, 11169-11186 (1996).

\section{Acknowledgments}

Fruitful discussions with C. Schüßler-Langeheine, F. M. F. de Groot and P. S. Miedema as well as support by E. Weschke and B. Klemke are gratefully acknowledged. We thank HZB for the allocation of synchrotron radiation beamtime. Funded by BMBF (05 ES3XBA/5) and DFG (WE2623/3-1). We are grateful to NSC under Swedish National Infrastructure for Computing (SNIC) and the PRACE-2IP project (FP7 RI-283493) resource Abel supercomputer based in Norway at University of Oslo for providing computing facility. B.S. acknowledges VR Swedish Research Links programme and Carl Tryggers Stiftelse for financial support. O.E. acknowledges support from the eSSENCE, VR, KAW foundation and the ERC.

\section{Author contributions}

D.S. and C.S.A. performed the measurements, the data evaluation and wrote the manuscript, A.W. performed the XMCD measurements, M.D. prepared the nanoparticles, S.H. and S.B. performed the calculations, O.E. and B.S. wrote the theoretical part of the manuscript, and H.W. wrote the manuscript.

\section{Additional information}

Supplementary information accompanies this paper at http://www.nature.com/ scientificreports

Competing financial interests: The authors declare no competing financial interests.

How to cite this article: Schmitz, D. et al. The dipole moment of the spin density as a local indicator for phase transitions. Sci. Rep. 4, 5760; DOI:10.1038/srep05760 (2014).

This work is licensed under a Creative Commons Attribution-NonCommercialNoDerivs 4.0 International License. The images or other third party material in this article are included in the article's Creative Commons license, unless indicated otherwise in the credit line; if the material is not included under the Creative Commons license, users will need to obtain permission from the license holder in order to reproduce the material. To view a copy of this license, visit http:// creativecommons.org/licenses/by-nc-nd/4.0/ 